\title{
Monte Carlo simulations of random copolymers at a selective
}

\author{
interface \\ Gongwen Peng , Jens-Uwe Sommer and Alexander Blumen \\ Theoretische Polymerphysik, Universität Freiburg, Rheinstr.12, D-79104 Freiburg, Germany
}

October 15, 2018

\begin{abstract}
We investigate numerically using the bond-fluctuation model the adsorption of a random AB-copolymer at the interface between two solvents. From our results we infer several scaling relations: the radius of gyration of the copolymer in the direction perpendicular to the interface $\left(R_{g z}\right)$ scales with $\chi$, the interfacial selectivity strength, as $R_{g z}=N^{\nu} f(\sqrt{N} \chi)$ where $\nu$ is the usual Flory exponent and $N$ is the copolymer's length; furthermore the monomer density at the interface scales as $\chi^{2 \nu}$ for small $\chi$. We also determine numerically the monomer densities in the two solvents and discuss their dependence on the distance from the interface.
\end{abstract}

PACS numbers: $61.25 . \mathrm{Hq}, 83.70 . \mathrm{Hq}$

*Permanent address: Institute of Physics, Academia Sinica, Beijing, China. 
Copolymers at interfaces are very important in technical applications. For example, the interface between two immiscible polymer melts can be mechanically reinforced by dissolving copolymers into the system [1, 2]. In other applications the surface tension between two immiscible solvents, e.g., oil and water, can be reduced by adding block copolymers consisting of a hydrophilic and a hydrophobic part. Thus the adsorption of copolymers at interfaces has received particular attention [3-7]. For diblock copolymers, the difference in the solubilities of the monomers favors the localization of the copolymer at the interface, with each block in its favorable solvent. However, for random copolymers frustrated situations may arise since the chain's connectivity forces some monomers to stay in their unfavourable solvent. Using a Hartree-type approach Garel et al [3] have studied the localization transition of an ideal random chain at an interface. Yeung, Balazs and Jasnow [4] have addressed the question of correlations in the A- and B-distributions, a point investigated recently through computer simulations by Balazs et al [7] in the context of copolymer brushes. In this paper we study numerically under excluded volume conditions a single random copolymer at an interface and we pay particular attention to scaling $[8]$.

We use the bond-fluctuation model (BFM) [9, 10] to perform Monte Carlo (MC) simulations. In the BFM the polymers obey the excluded-volume requirements, and the motion occurs such that the bonds do not cross each other, see Refs. 9, 10 for details. Here we take a cubic box of size $L \times L \times H$, with periodic boundary conditions in the $\mathrm{x}-$ and $\mathrm{y}$-directions and two impenetrable surfaces at $z=0$ and $z=H$. We study the behavior of a single copolymer consisting of $N$ randomly chosen monomers of A- and of B-type. We assume a symmetrical situation: the interaction parameter of the monomers is $\chi k_{B} T$ when immersed in their unfavorable solvent and zero otherwise. The solvent below the interface $(z \leq H / 2)$ favors A-type monomers, and the solvent above the interface $(z \geq H / 2+1)$ B-type monomers. Note that the interface is thus at $z=(H+1) / 2$. In each Monte Carlo step the chain moves by position changes of the monomers, which attempt nearest neighbor steps on the underlying cubic lattice. A move is taken into consideration only if it satisfies the requirements of self-avoidance and of non-crossing of bonds. Furthermore, energetically unfavorable moves are statistically permitted according to the usual Boltzmann factor.

We obtain results for copolymers of lengths $N=16,32,64$, and 128 using systems with sizes 
$L=50$ and $H=100$. An initial configuration is generated starting with the first monomer near the interface and then randomly adding the subsequent monomers such that self-avoidance and non-crossing of bonds are obeyed. The energetic aspects of the interaction with the solvents are then taken care of by the usual Boltzmann factor; the monomer-monomer interaction is only accounted for through the excluded volume aspects. We let the chain move for a long time according to the MC prescriptions, such that the chain relaxes to equilibrium. The averaged quantities which we will show below are then obtained from such equilibrium configurations. We found numerically the relaxation time (determined using the autocorrelation function of the radius of gyration $R_{g}$ and of its z-component $R_{g z}$ [1]) to be around 50,000 Monte Carlo steps (MCS), where a MCS consists of $N$ move attempts; we thus view the copolymer as having reached equilibrium after 200,000 MCS. Averages are then calculated from the configurations obtained in the subsequent 200,000 MCS. For each copolymer length we realized 100 independent runs.

Figure 1 shows the normalized probability to find monomers of A-type and of B-type at the height $z$. In this example the copolymer length is $N=128$ and we have chosen $\chi=3.15$. Apart from the (expected) symmetry between the plotted curves, the figure shows that most of the monomers are located near the interface; the copolymer is thus adsorbed. Each of the two curves peaks near the interface, on the favorable side. The curves decay smoothly on the favorable side and sharply across the selective interface. There is evidence of frustration in the form of a secondary peak in monomer density on the unfavorable solvent side of the interface. This situation arises because the covalent bonding of the chain forces some monomers (whose neighbors are in majority of the other type) to be immersed in the "wrong" solvent. This situation is due to an energetic-entropic balance: configurations with monomers in the "wrong" solvent are energy-unfavorable but favorable in view of entropy.

Evidently, in Fig. 1 the asymmetry of each curve with respect to the interface is related to the value of $\chi$, a fact reminiscent of paramagnetism, where applying an external field increases (decreases) the numbers of spins parallel (antiparallel) to it. Here we determine the magnitude of the asymmetry across the interface by evaluating 


$$
M=\sum_{z=0}^{H}\left|\rho_{A}(z)-\rho_{B}(z)\right|=\sum_{z=0}^{H / 2} \rho_{A}(z)-\sum_{z=H / 2+1}^{H} \rho_{A}(z)+\sum_{z=H / 2+1}^{H} \rho_{B}(z)-\sum_{z=0}^{H / 2} \rho_{B}(z) .
$$

Figure 2 shows the relation between $M$ and $\chi$ for different copolymer lengths $N$. Note the very small dependence of $M$ on $N$; the curves for different $N$ almost coincide. As in paramagnetism, $M$ is a linear function of $\chi$ for small $\chi$, and reaches a constant (here unity due to normalization) for large $\chi$.

We now turn to the question how the copolymer behaves around the interface. For this we compute $R_{g z}$, the $\mathrm{z}^{-}$component of the radius of gyration of the copolymer. Here $R_{g z}^{2}=\sum_{i=1}^{N}\left(z_{i}-\bar{z}\right)^{2} / N$, where $z_{i}$ is the $\mathrm{z}$-component of the $i$ th monomer's position and $\bar{z}=\sum_{i=1}^{N} z_{i} / N$. We are interested in the dependence of $R_{g z}$ on $\chi$ and hence display in Fig. $3 R_{g z}(\chi) / R_{g z}(0)$ as a function of $\sqrt{N} \chi$ for different $N$. Notice that (except for very large $\chi$ ) all data collapse into a single curve. This demonstrates that $R_{g z}(\chi)$ scales with $\sqrt{N} \chi$ for small and moderately large $\chi$. Scaling fails when $\chi$ is very large, because then the copolymers are practically squeezed on the interface, having the AB covalent bonds at the interface. In the region in which the curves collapse we observe a constant regime for very small $\chi$ followed by a power-law decay regime for moderate $\sqrt{N} \chi$ values. Setting $R_{g z}(\chi) / R_{g z}(0)=f(\sqrt{N} \chi)$ with $f(y)=1$ for $y<y_{c}$ and $f(y)=y^{-\alpha}$ for $y>y_{c}$, we find from Fig. 3 that the crossover value $y_{c}$ is roughly $y_{c} \approx 10$. Furthermore, from a best fit to the data in the powerlaw regime we obtain numerically for the exponent $\alpha$ that $\alpha=1.112 \pm 0.10$ i.e., $\alpha / 2=0.56 \pm 0.05$. This result is in agreement with the scaling arguments of Refs. [6, 12, which predict that $\alpha$ should equal $2 \nu$, where $\nu$ is the usual Flory exponent, $\nu \approx 0.588$ for excluded-volume chains. Scaling with $\sqrt{N} \chi$ is consistent with the "blob"-picture of a random copolymer at an interface [6, 12]. A blob is a chain segment containing $g(g \gg 1)$ monomers, and thus has roughly $g^{1 / 2}$ monomers of one type in excess; the total number of blobs is $N / g$ and their number at the interface depends on $\sqrt{N} \chi[12$.

Another example of scaling is provided by the monomer density exactly at the interface $z=$ $(H+1) / 2$. In our (discrete) lattice model we thus compute

$$
\rho_{s}=\frac{1}{2}\left[\rho_{A}\left(\frac{H}{2}\right)+\rho_{B}\left(\frac{H}{2}+1\right)\right] .
$$

In Fig. 4 we display in a $\log -\log$ plot $\rho_{s}$ as a function of $\chi$ for different polymer lengths $N$. Again the data for different $N$ coincide, possibly with the exception of the very small $\chi$ regime; here, 
however, $\rho_{s}$ is small and thus the relative error is rather large. For very large $\chi, \rho_{s}$ gets to be independent of $\chi$, as the chains are then squeezed on the surface. In the moderate $\chi$ regime $\rho_{s}$ scales with $\chi$ as a power-law $\rho_{s} \sim \chi^{\beta}$, which is illustrated by the linearity of the data in the $\log -\log$ plot of Fig. 4. From a best fit to the data in this regime we obtain numerically that the exponent $\beta$ is $1.14 \pm 0.06$. This result can be compared to the expression $\beta=2 \nu$ in the "blob" picture [6, 12], i.e., taking the accepted $\nu \approx 0.588$ for excluded volume chains to $\beta \approx 1.176$. The derivation of $\beta=2 \nu$ according to Refs. [6] and [12] starts from the scaling of physical variables with $N^{1 / 2} \chi$. Hence the total number of monomers at the interface obeys $N_{s}=N^{1-\nu} f\left(N^{1 / 2} \chi\right)$, where $f(0)=1$. Noting that for large $\chi N_{s}$ is proportional to $N$ requires that $f(y) \sim y^{m}$, with $1-\nu+m / 2=1$. Hence $m=2 \nu$ and $N_{s} \sim N \chi^{2 \nu}$. Due to normalization one finds $\rho_{s}=N_{s} / N \sim \chi^{2 \nu}$.

We conclude by showing that even the total monomer density scales with $\chi^{2 \nu}$. In Fig. 5(a) we plot $\left(\rho_{A}(z)+\rho_{B}(z)\right) / \rho_{0}$ as a function of $\left(z-z_{0}\right) \chi^{2 \nu}$ with $z_{0}=(H+1) / 2$ and $\rho_{0}=\frac{1}{2}\left[\rho_{A}\left(\frac{H}{2}\right)+\rho_{B}\left(\frac{H}{2}\right)+\right.$ $\left.\left.\rho_{A}\left(\frac{H}{2}+1\right)+\rho_{B}\left(\frac{H}{2}+1\right)\right]\right)$. For a series of $N$ and $\chi$ values we find that all data collapse to a single curve when $\chi$ lies in the power-law regime of Fig. 3. This implies the scaling relation

$$
\left(\rho_{A}(z)+\rho_{B}(z)\right)=\rho_{0} g\left(\left(z-z_{0}\right) \chi^{2 \nu}\right)
$$

Eq. (3) does not hold when $\chi$ lies outside the power-law regime. Fig. 5(b) displays the data of Fig. 5(a) in a semi-logarithmic plot. The appearance of two almost straight lines rules out a Gaussian behavior and suggests to approximate the wings of $g(y)$ by an exponential form. We find for the wings $g(y) \sim \exp (-\gamma|y|)$, with $\gamma=0.10 \pm 0.02$.

In conclusion, we have studied numerically the adsorption of random copolymers at a selective interface. The main results are as follows. For small and for moderately large $\chi$ the magnitude $M$ of the asymmetry, as defined in Eq. (1), grows linearly with $\chi$ and is independent of $N$. Furthermore, $R_{g z}$, the radius of gyration in the direction perpendicular to the interface scales as $R_{g z}=N^{\nu} f(\sqrt{N} \chi)$. The monomer density $\rho_{s}$ at the interface, Eq. (3), scales as $\chi^{2 \nu}$; moreover, for $\chi$ in the power-law regime the total monomer density obeys $\rho_{A}(z)+\rho_{B}(z)=\rho_{0} g\left(\left|z-z_{0}\right| \chi^{2 \nu}\right)$ where $g(y)$ is close to being exponential. 
This work was supported by the Deutsche Forschungsgemeinschaft (SFB 60), by the Fonds der Chemischen Industrie and by PROCOPE, administrated by the DAAD. GP thanks the Alexander von Humboldt Foundation for a fellowship. 


\section{References}

[1] H. R. Brown, V. R. Deline and P. F. Green, Nature (London) 341, 221 (1989).

[2] C.-A. Dai, B.J. Dair, K. H. Dai, C. K. Ober, E. J. Kramer, C.-Y. Hui and L. W. Jelinski, Phys.Rev.Lett. 73, 2472 (1994).

[3] T. Garel, D. A. Huse, S. Leibler and H. Orland, Europhys.Lett. 8, 9 (1989).

[4] C. Yeung, A. C. Balazs and D. Jasnow, Macromolecules 25, 1357 (1992).

[5] A. Grosberg, S. Izrailev and S. Nechaev, Phys.Rev. E 50, 1912 (1994).

[6] J. -U. Sommer and M. Daoud, Europhys. Lett., in press (1995).

[7] A. C. Balazs, D. Gersappe, R. Israels and M. Fasolka, Macromol.Theory Simul. 4, 585 (1995); D. Gersappe, M. Fasolka, R. Israels and A. C. Balazs, Macromolecules 28, 4753 (1995).

[8] P.G. de Gennes, Scaling Concepts in Polymer Physics (Cornell University Press, Ithaca and London, 1979).

[9] I. Carmesin and K. Kremer, Macromolecules 21, 2819 (1988).

[10] H. P. Deutsch and K. Binder, J. Chem. Phys. 94, 2294 (1991).

[11] P.-Y Lai and K. Binder, J. Chem. Phys. 95, 9288 (1991).

[12] J. Sommer, G. Peng and A. Blumen, submitted (1995). 


\section{Figure Captions}

Figure 1: The probability (normalized density) $\rho$ to find a monomer of A-type (diamonds) or B-type (crosses) at height $z$. Here the copolymer length is $N=128$ and $\chi=3.15$.

Figure 2: The dependence of the asymmetry parameter $M$, Eq.(1) on $\chi$ for different polymer lengths.

Figure 3: $R_{g z}(\chi) / R_{g z}(0)$ as a function of the scaling variable $\sqrt{N} \chi$ for different polymer lengths.

Figure 4: The density $\rho_{s}$ exactly at the interface versus the interfacial selectivity strength $\chi$ for different polymer lengths.

Figure 5(a): The total monomer density plotted as a function of $\left(z-z_{0}\right) \chi^{2 \nu}$, see text for details. The parameter values are $a: N=32, \chi=3.15 ; b: N=64, \chi=2.20 ; c: N=64, \chi=3.15 ; d$ : $N=128, \chi=1.55 ; e: N=128, \chi=2.30 ; f: N=128, \chi=3.15$.

Figure 5(b): Same as (a) but plotted semi-logarithmically. 


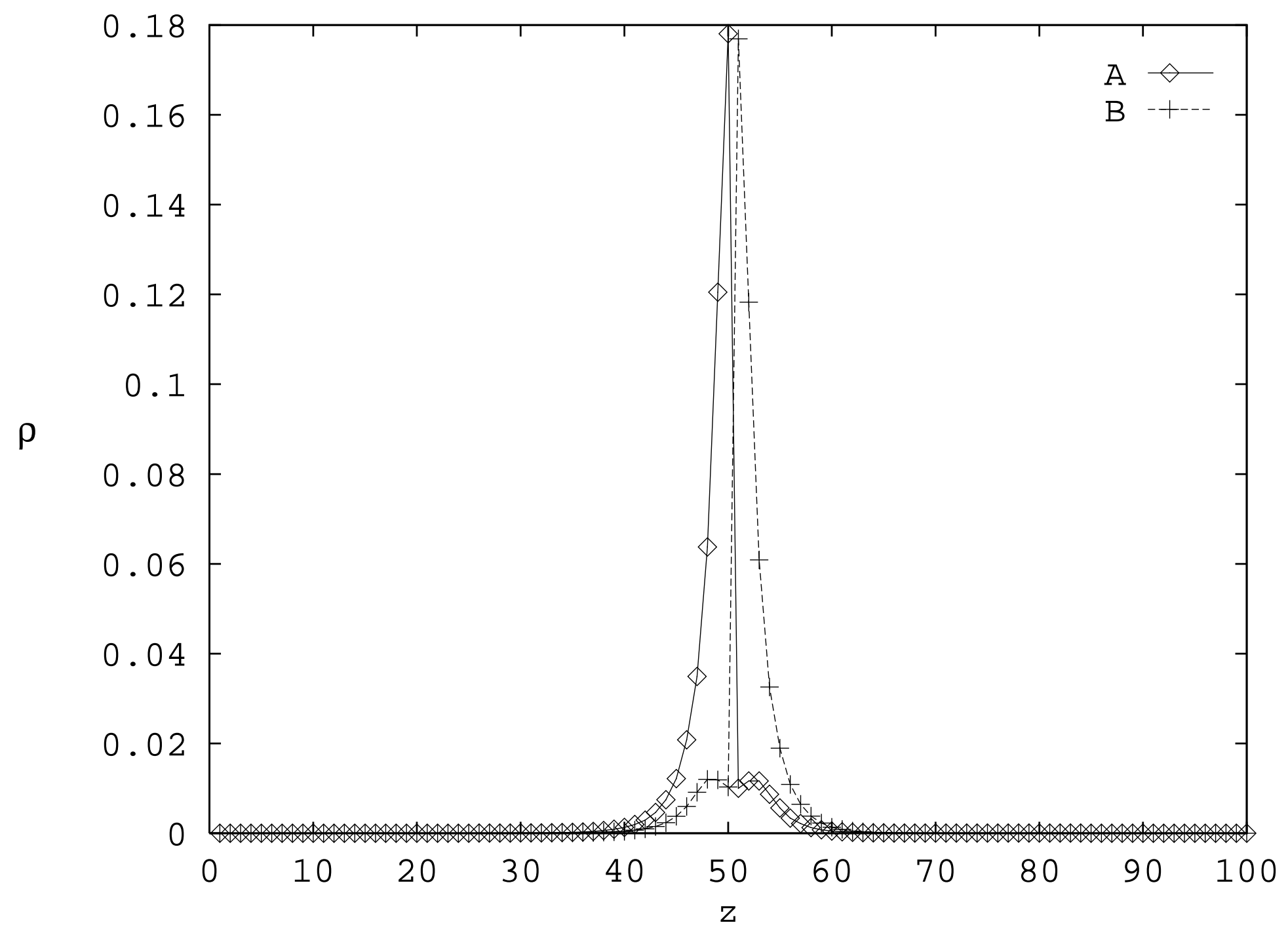




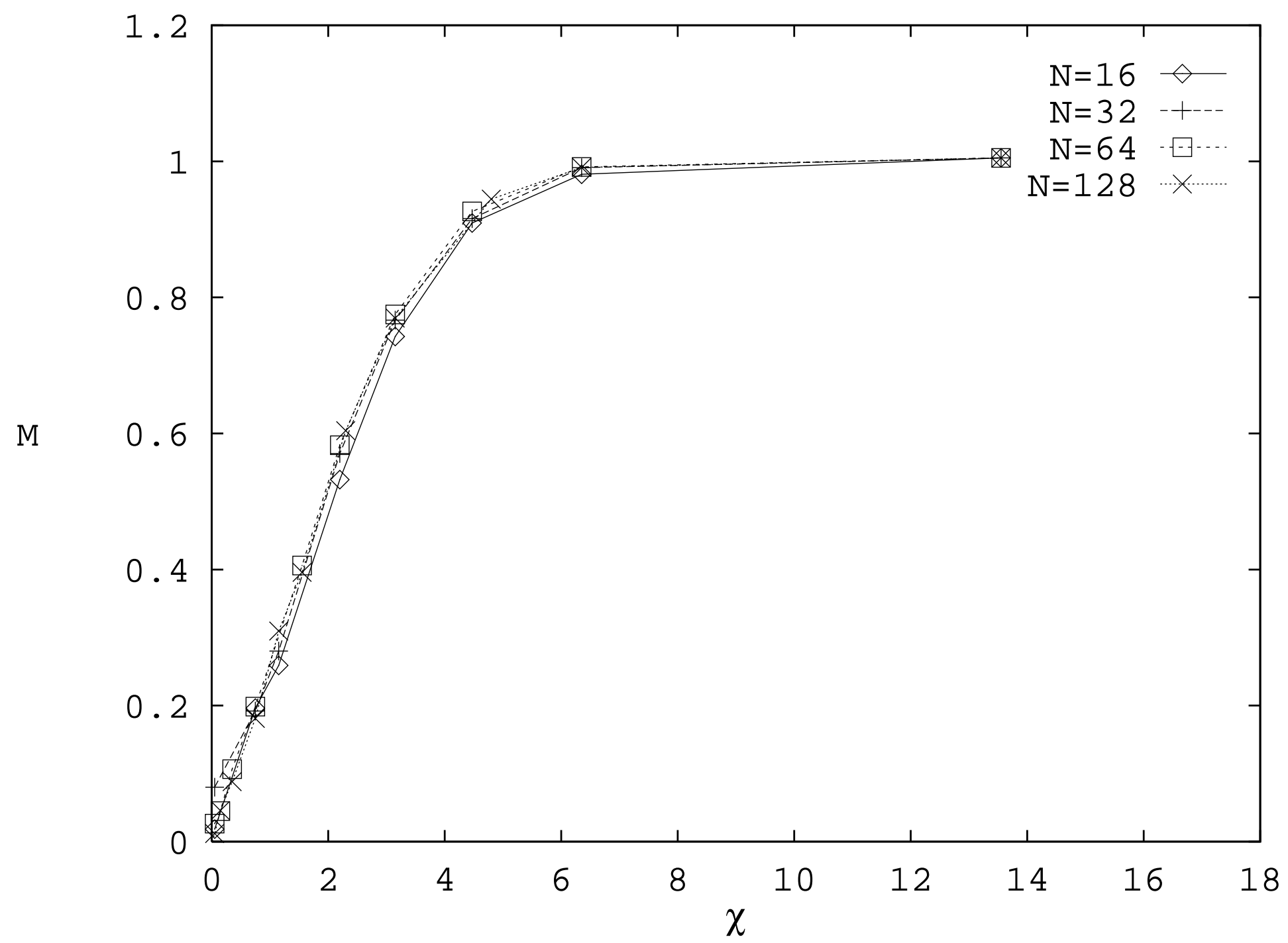




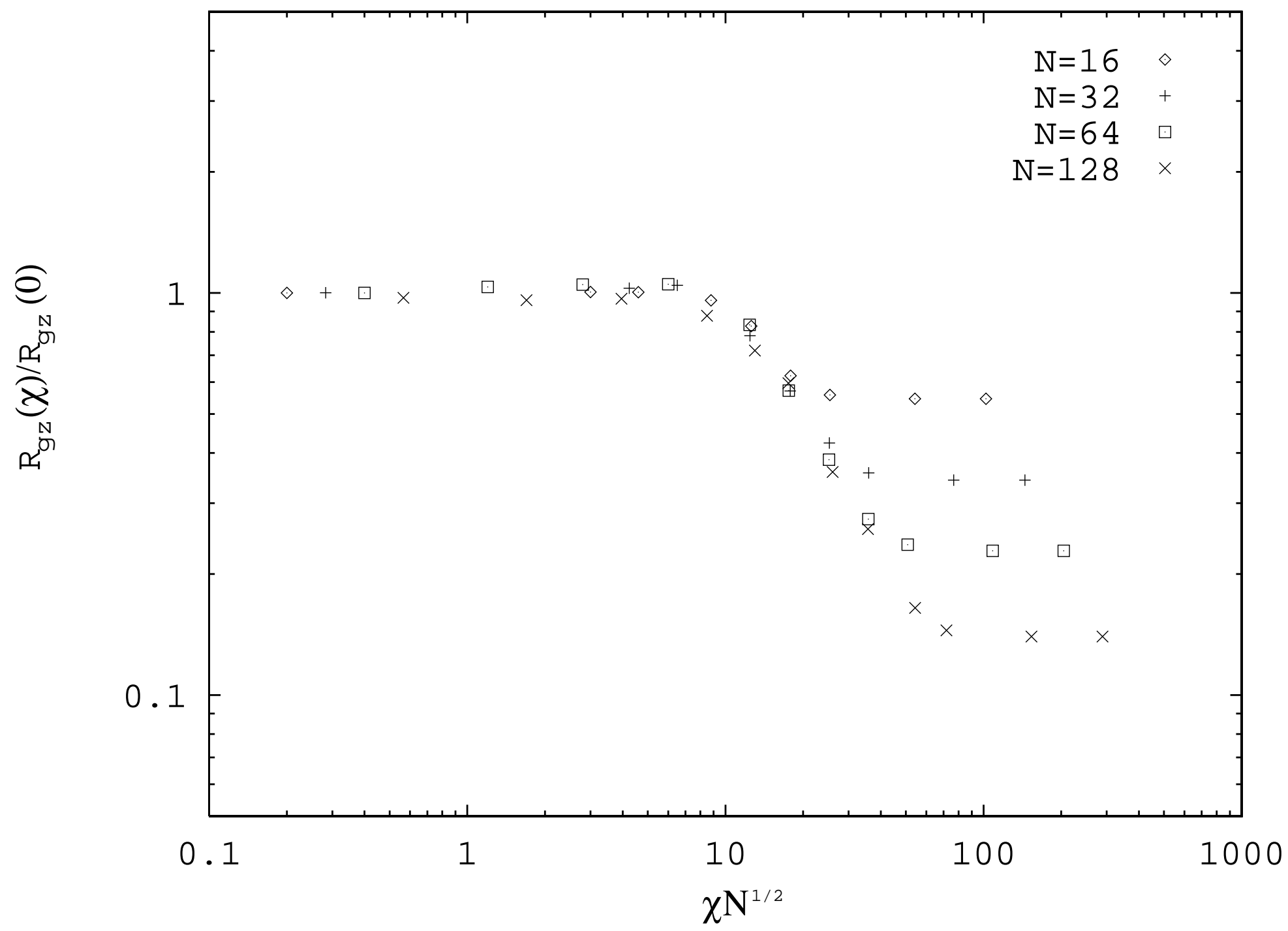




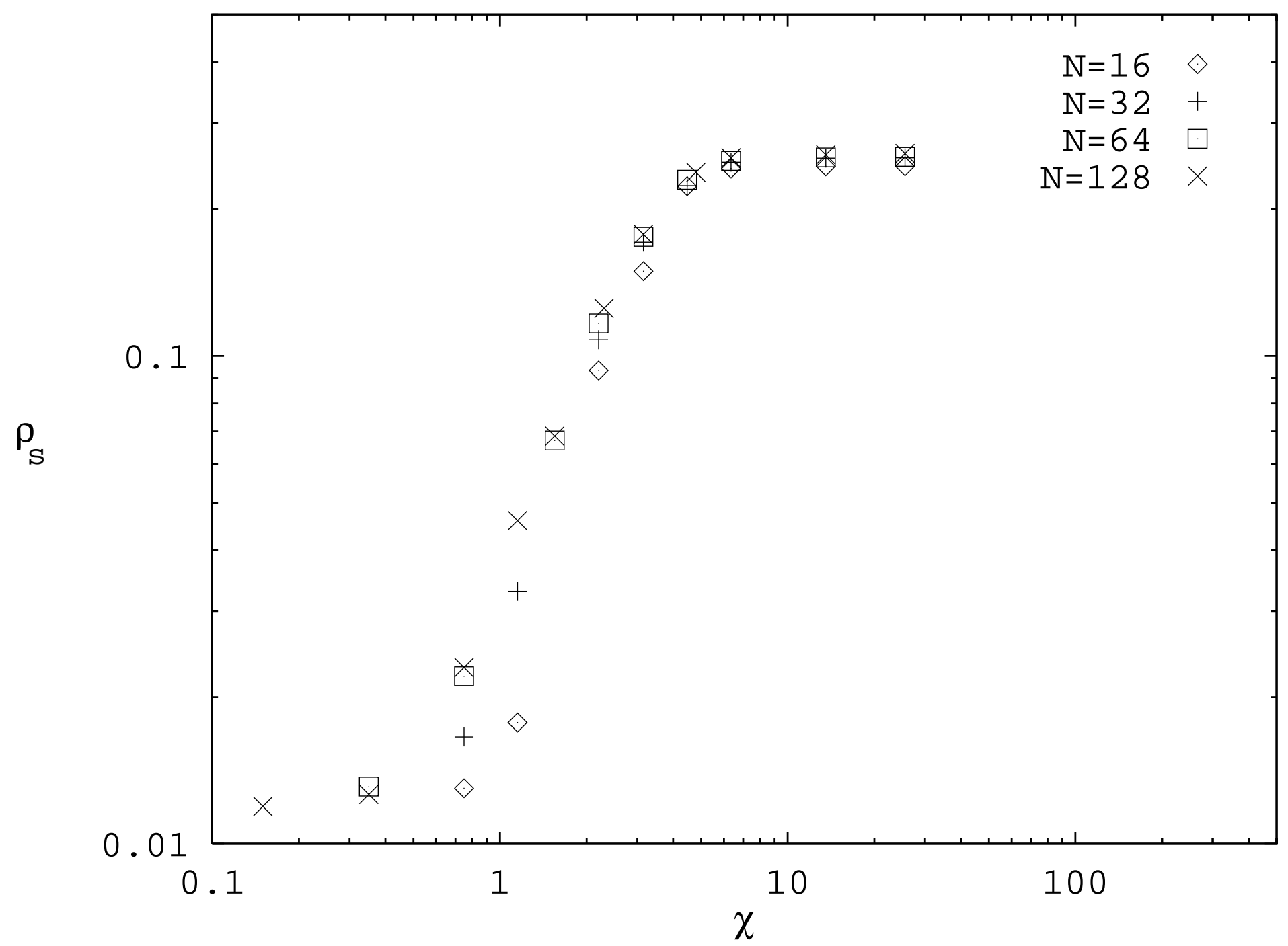




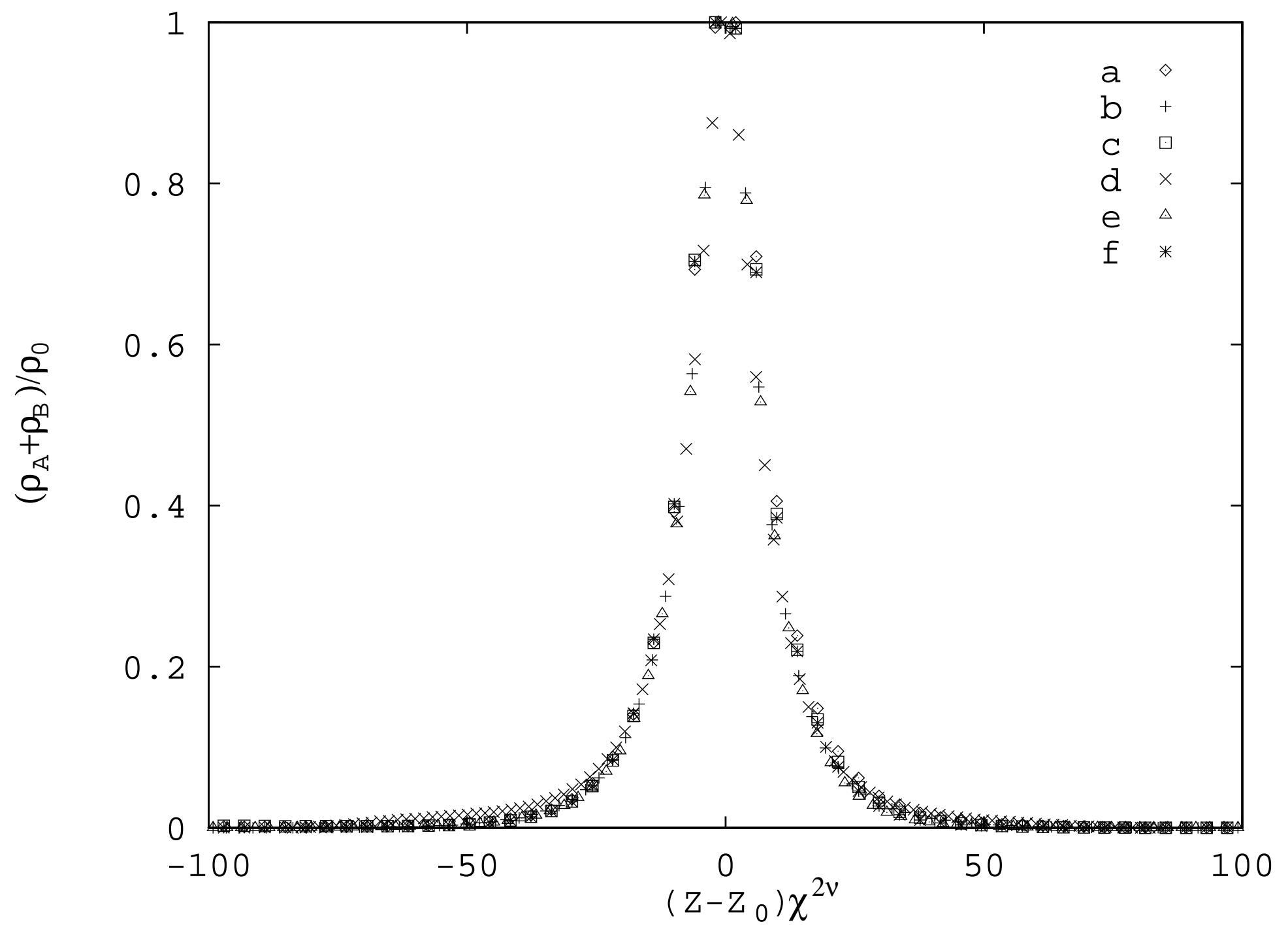




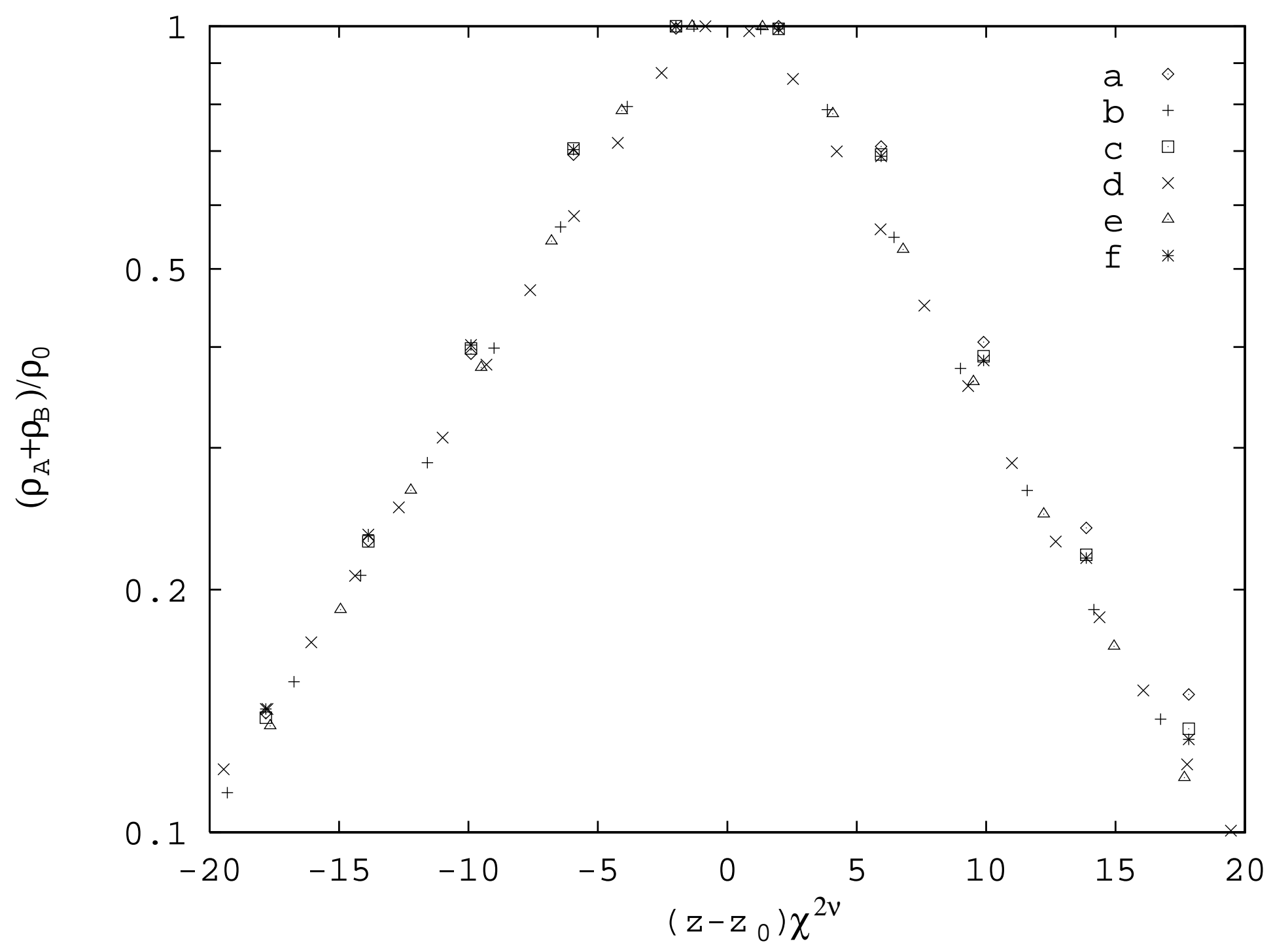

